\title{
SHEAR-INDUCED INFLATION OF CORONAL MAGNETIC FIELDS
}

James A. Klimchuk

CSSA-ASTRO-89-11

August 5, 1989

NBL 05.020.272 


\begin{abstract}
Using numerical models of force-free magnetic fields, we have examined how the shearing of footpoints in arcade geometries leads to an inflation of the coronal magnetic field. For each of the shear profiles considered, all of the field lines become elevated compared with the potential field. This includes cases where the shear is concentrated well away from the arcade axis, such that $B_{z}$, the component of field parallel to the axis, increases outward to produce an inward $\mathrm{B}_{\mathrm{z}} 2 / 8 \pi$ magnetic pressure gradient force. These results contrast with an earlier claim, shown to be incorrect, that field lines can sometimes become depressed as a result of shear. We conjecture that an inflation of the entire field will always result from the shearing of simple arcade configurations. These results have implications for prominence formation, the interplanetary magnetic flux, and possibly also coronal holes.
\end{abstract}

$$
\begin{aligned}
& \text { Estoz)siand } 1 / 8 \text { i } \\
& \text { l) }
\end{aligned}
$$




\section{INTRODUCTION}

A majority of observed solar phenomena are either caused by or significantly influenced by the magnetic fields which permeate the solar atmosphere. Magnetic fields give structure to the atmosphere; they direct the flow of mass and many forms of energy; they may be an important source of coronal heating; and they power many of the most spectacular solar events, most notably solar flares. It is not surprising, therefore, that considerable effort has been devoted to studying the properties of solar magnetic fields.

Deep within the photosphere and below, the magnetic field is dominated by the highly massive and reasonably highly conducting gas in which it is embedded. Any motions of the gas will cause the magnetic field to be dragged about the solar surface. In the overlying corona, on the other hand, the gas density and pressure are sharply reduced and magnetic forces tend to greatly exceed those associated with the material. In this situation the Lorentz force must very nearly vanish (for equilibrium), and it is therefore useful to study the properties of so-called force-free magnetic fields, for which

$$
(\nabla \times \mathbf{B}) \times \mathbf{B}=0 .
$$

One important property of these fields is their energy content. As photospheric and subphotospheric motions displace magnetic footpoints, electric currents are induced in the corona. The energy associated with these currents is known as the "free magnetic energy" and is energy which can liberated from the field in the form of a flare, for example. Klimchuk, Sturrock, and Yang (1988) have recently shown that systematic shearing motions in arcade configurations will produce energy increases that can be simply and predictably described. 
Another important property of force-free magnetic fields is their structure, and in particular, the manner in which this structure changes as the footpoint positions are changed. More than 20 years ago, Sturrock and Woodbury (1967) demonstrated that magnetic field configurations can become inflated as the footpoints are sheared; that is, the field lines rise and spread out horizontally. A number of later calculations revealed this to be a common behavior (e.g., Barnes and Sturrock 1972; Low 1977; Jockers 1977; Birn, Goldstein, and Schindler 1978; Priest and Milne 1980; Yang, Sturrock, and Antiochos 1986; Zwingmann 1987; Mikic, Barnes, and Schnack 1988; Klimchuk, Sturrock, and Yang 1988; Biskamp and Welter 1989; Klimchuk and Sturrock 1989; Finn and Chen 1989; Amari et al. 1989). This is not an unexpected result, since shearing motions increase the strength of the field, and the associated increase in magnetic pressure, which is equivalent to an increase in thermal gas pressure (see below), should cause an overall expansion of the configuration.

We are aware of at least one calculation, however, where an opposite behavior seems to occur. In one of two cases studied by Low and Nakagawa (1975), it was reported that the field becomes depressed (i.e., the field lines descend) as the shear of the footpoints increases. This is an intriguing and potentially very important result that has received considerable attention in the review literature (e.g., Birn and Schindler 1981; Priest 1982a; Low 1982).

There are a variety of reasons for wanting to study how magnetic configurations change in response to footpoint shearing motions. As configurations become inflated, for example, closed field lines are brought to greater heights in the atmosphere, where they are more likely to be opened up to become part of the outflowing solar wind (e.g., Pneuman and Kopp 1971). This has consequences for the interplanetary magnetic field, since as more 
field lines open, the quantity of interplanetary magnetic flux increases (see, for instance, Wang and Sheeley 1988). It is possible that this process could lead to the formation or growth of coronal holes. In addition, the lateral expansion of inflating configurations will tend to promote flux linkages between different parts of the Sun, since oppositely directed field lines will be forced together and hence will be more likely to reconnect.

The depression of magnetic field configurations may ultimately lead to prominence formation, since prominences cannot easily form unless there is a dip in the field (i.e., a region that is concave upward), or at the very least a region that is exceptionally flat (see the discussions in Priest, Hood, and Anzer 1989 and Amari et al. 1989). Even then, the hydrodynamics of prominence formation is not well understood (e.g., Poland, Mariska, and Klimchuk 1986). It is therefore important to fully understand the result of Low and Nakagawa (1975) and to determine what types of situations will, in general, lead to shearinduced depression rather than shear-induced inflation of force-free magnetic field configurations. We report here the details of our investigation into this problem (Klimchuk 1989).

\section{FOOTPOINT SHEAR AND MAGNETIC PRESSURE}

In many situations, the coronal magnetic field has an arcade-like geometry in which the field is approximately uniform in one of the horizontal directions. Active regions, for example, tend to have two dominant regions of opposite polarity separated by a long, though not always straight, neutral line (or, more properly, a "weak field corridor" where the flux is very small; Klimchuk 1987). Similarly, much the quiet Sun is divided into large, predominantly unipolar regions of opposite polarity that are stretched out into elongated patterns by a combination of supergranular diffusion, differential 
rotation, and meridional flow (see, for instance, Sheeley, Nash, and Wang 1987). In both situations, the overlying corona is characterized by nested loop systems referred to as arcades.

Often, the length of the arcade is substantially greater than its width, and it is therefore common to model magnetic field configurations that are assumed to have translational symmetry (i.e., to be infinitely long). Taking the $\mathrm{y}$-direction to be the vertical direction, with $\mathrm{y}=0$ corresponding to the photosphere, and the z-direction to be the direction of invariance, we can express the magnetic field in the form

$$
\mathbf{B}(\mathrm{x}, \mathrm{y})=\left(\frac{\partial \mathrm{A}}{\partial \mathrm{y}},-\frac{\partial \mathrm{A}}{\partial \mathrm{x}}, \mathrm{B}_{\mathrm{z}}\right) \text {, }
$$

which follows directly from the divergence-free nature of $\mathbf{B}$. Since

$$
\text { B } \cdot \nabla \mathrm{A}=0 \text {, }
$$

we see that the scalar function $A(x, y)$, which is simply the $z$-component of the magnetic vector potential, is constant along field lines. Contours of constant A in the $\mathrm{x}-\mathrm{y}$ plane are simply the projections of field lines onto that plane, and correspond to an end-on view of the arcade.

If we include the effects of gas pressure, the force balance equation [equation (1.1)] becomes

$$
\frac{1}{4 \pi}(\nabla \times B) \times B-\nabla P=0 .
$$


Upon substituting for $\mathbf{B}$ using equation (2.1), the z-component of force balance leads to the result that

$$
\mathrm{B}_{z}=\mathrm{B}_{z}(\mathrm{~A})
$$

so that $B_{z}$ is also constant along field lines (e.g., Priest 1982a). The ycomponent of force balance leads to

$$
\frac{1}{4 \pi} \nabla^{2} \mathrm{~A}+\frac{\mathrm{d}}{\mathrm{dA}}\left(\mathrm{P}+\frac{1}{8 \pi} \mathrm{B}_{\mathrm{z}}^{2}\right)=0
$$

which is the well-known Grad-Shafranov equation. It shows explicitly that the magnetic pressure associated with the z-component of the magnetic field is equivalent to gas pressure in the way that it affects the equilibrium distribution of field lines, $A(x, y)$. Note that if the gravitational scale height is large compared with the geometric height, so that $\mathrm{P}$ does not depend explicitly on height, then it too is constant along field lines. Having established that $B_{z}$ and $P$ are analogous in this sense, we henceforth concern ourselves only with force-free magnetic fields, for which $P=0$. It is important to point out, however, that the choice $B_{z}(A)$ is not as flexible as the choice $P(A)$ from a physical point of view (e.g., Klimchuk and Sturrock 1989).

In an unsheared magnetic arcade, which is a potential field, all field lines lie in planes of constant $z$ and $B_{z}=0$. By shearing the field (that is, by displacing the footpoints of one or more field lines in the z-direction so that the two footpoints of a given field line are separated in $z$ ), we introduce a finite $B_{z}$ component. We expect the additional magnetic pressure associated with this component to produce an overall inflation of the field; although, in principle, 
some parts of the field could become depressed. This expected inflation has been found to occur in a large number of specific arcade models, as cited in the Introduction [see also Wolfson and Gould (1985) and Hundhausen, Hundhausen, and Zweibel (1981) for a discussion of inflated fields in a spherical geometry].

Suppose that the distribution of shear is such that $B_{z}$ increases outward from the arcade axis and reaches a maximum value a finite distance away from the axis. Then there will be both inward and outward $\mathrm{B}_{\mathrm{z}}^{2}$ pressure gradient forces--inward in the inner part of the arcade, and outward in the outer part. One might expect those field lines which lie underneath the field line of maximum $B_{z}$ to become depressed, and those field lines which lie above it to become elevated. This would seem to be the situation in which depressed field is most likely to occur, and is the situation we wish to explore.

One way to calculate force-free magnetic fields is to specify the functional dependence of $B_{z}$ upon $A$ explicitly, which we refer to as the "generating function" method. In principle, it is then straightforward to study cases where $B_{z}$ is maximized away from the arcade axis. Analytic solutions are often difficult to obtain, however, and only very recently have numerical solutions with this type of $\mathrm{B}_{\mathrm{z}}$ dependence been calculated (Amari et al. 1989). Moreover, solutions obtained by the generating function method can sometimes have peculiar, unphysical properties, as discussed in Section $I V$ and in Klimchuk and Sturrock (1989). It generally agreed that it is physically more reasonable to define the magnetic field in terms of footpoint boundary conditions, i.e., the shear, than it is to place global constraints on $B_{z}$ (e.g., Sturrock and Woodbury 1967; Jockers 1977; Low 1982; Priest 1982a,b; Aly 1985; Amari et al. 1989; Finn and Chen 1989; Klimchuk and Sturrock 1989). This is the approach that we take. 
The relationship between $B_{z}$ and shear is not always a simple one. For instance, it is not generally true that the field lines with the greatest footpoint separation (in $\mathrm{z}$ ) are also the field lines with the greatest $\mathrm{B}_{\mathbf{z}}$. To see this, let $\mathbf{s}$ be the spatial coordinate along a contour of $\mathrm{A}$. We then have the relationship

$$
\frac{\mathrm{dz}}{\mathrm{B}_{\mathrm{z}}}=\frac{\mathrm{ds}}{\mathrm{B}_{\perp}}
$$

where $B_{\perp} \equiv|\nabla A|$ is the magnitude of the projection of the magnetic field vector onto the $x-y$ plane. We can integrate along the contour to get the $z$ separation between the two footpoints:

$$
\Delta \mathrm{z}=\int_{\mathrm{A}} \frac{\mathrm{B}_{\mathrm{z}}}{\mathrm{B}_{\perp}} \mathrm{ds} .
$$

Noting that $B_{z}$ is constant on $A$, we have

$$
\mathrm{B}_{\mathrm{z}}=\left\langle\mathrm{B}_{\perp}^{-1}\right\rangle^{-1} \mathrm{~L}^{-1} \Delta \mathrm{z}
$$

where $L$ is the length of the contour. Thus, $B_{z}$ is related not only to the footpoint separation, but also to the mean strength and length of the projected field line. For a shear profile in which $\Delta \mathrm{z}$ increases away from the neutral line, it does not necessarily follow that $B_{z}$ will also increase, since both $\left\langle B_{\perp}^{-1}\right\rangle^{-1}$ and $\mathrm{L}^{-1}$ decrease. How $\mathrm{B}_{\mathrm{z}}$ varies in space depends on the details of the particular model. 


\section{MODEL CALCULATIONS}

Equation (2.1) is one mathematical representation of magnetic fields having translational symmetry. Another makes use of Clebsch variables $\alpha$ and $\beta$ :

$$
\mathbf{B}=\nabla \alpha \times \nabla \beta
$$

It is clear that $\alpha$ and $\beta$ are constant along field lines. Furthermore, it can be easily shown that $\alpha$ is equivalent to $A$ in equation (2.1). It is convenient to adopt the form

$$
\alpha=\alpha(x, y), \quad \beta=z-\gamma(x, y),
$$

because then $\gamma$ measures the displacement of any field line in the z-direction (Sturrock and Woodbury 1967). By specifying $\alpha$ and $\gamma$ as functions of $x$ on the plane $y=0$ we are able to specify both the normal value of the photospheric magnetic field and the field line connectivity (i.e., the relative positions of the magnetic footpoints).

We solve equation (1.1) subject to these boundary conditions using a numerical relaxation technique known as the "magneto-frictional method" (Yang, Sturrock, and Antiochos 1987; Klimchuk, Sturrock, and Yang 1988; Klimchuk and Sturrock 1989). Basically, an initial guess at the coronal field relaxes to a force-free state through motions that are proportional to the local Lorentz force. The constant of proportionality can be interpreted as a frictional force, which is the origin of the method's name. Interested readers are referred to the above references for more details (see also Chodura and Schluter 1981 and Craig and Sneyd 1986). 
For photospheric boundary conditions on $\alpha$ we use the normal field distribution advocated by Klimchuk, Sturrock, and Yang (1988) as being appropriate for solar active regions:

$$
B_{y}(x, 0)=\frac{x}{|x|}\left\{\begin{array}{ll}
0 & |x| \leq c \\
\sin \left[(|x|-c) \frac{\pi}{2}\right] & c<|x| \leq c+1 \\
1 & c+1<|x| \leq c+d+1 \\
\sin \left[(|x|-c-d) \frac{\pi}{2}\right] & c+d+1<|x| \leq c+d+2 \\
0 & |x|>c+d+2
\end{array}\right\},
$$

so that

$$
\alpha(x, 0)=\frac{x}{|x|}\left\{\begin{array}{ll}
d+\frac{4}{\pi} & |x| \leq c \\
\frac{2}{\pi} \cos \left[(|x|-c) \frac{\pi}{2}\right]+d+\frac{2}{\pi} & c<|x| \leq c+1 \\
-x+c+d+1+\frac{2}{\pi} & c+1<|x| \leq c+d+1 \\
\frac{2}{\pi} \cos \left[(|x|-c-d) \frac{\pi}{2}\right]+\frac{2}{\pi} & c+d+1<|x| \leq c+d+2 \\
0 & |x|>c+d+2
\end{array}\right\} .
$$

Here, $\mathrm{c}$ is the half-width of the "weak field corridor" found to separate the opposite polarity parts of most active regions (Klimchuk 1987), and $d+2$ is the width of the individual strong field regions, or "plages." We choose $c=1$ and $d$ $=4$, in which case the units of our spatial coordinates correspond to roughly $10^{4} \mathrm{~km}$ on the Sun. The normal field distribution $B_{y}$ is shown in Figure 1. 
To guarantee that $B_{z}$ has its maximum a finite distance away from the arcade axis we adopt the following localized shear profile:

$$
\gamma(x, 0)=Z_{\max } \exp \left[-\left(x-x_{0}\right)^{2}\right]
$$

where $x_{0}=4$. This shear profile is also shown in Figure 1. $B_{z}$ is greatest for the $\alpha=0.62$ field line, which is displaced inward somewhat from the field line of maximum shear ( $\alpha=0.5$ ), as expected on the basis of equation (2.8).

The numerical computations are performed on a finite-difference grid covering the domain $0 \leq \mathrm{x} \leq 60,0 \leq \mathrm{y} \leq 60$ (only half the field need be computed explicitly due to symmetry about the $\mathrm{x}=0$ plane). A total of $289 \times 289$ grid points are placed nonuniformly throughout this domain to provide a resolution of 0.11 near the origin and 0.51 at large distances. "Superconductor" boundary conditions $(\alpha=0)$ at the outer boundaries $\mathrm{x}=60$ and $\mathrm{y}=60$ prevent flux from leaving the box, but the boundaries are far enough removed that they have negligible effect on the field near the source.

Contours of constant $\alpha$ are shown in Figure 2a for the potential field case $\mathrm{Z}_{\max }=0$ and in Figure $2 \mathrm{~b}$ for the sheared field case $\mathrm{Z}_{\max }=6$. It is clear from a comparison of the plots and from an examination of the raw model data that all of the field lines are elevated in the sheared case. Thus, our initial expectations that the inner field lines might become depressed were incorrect.

We have examined several other shear functions to determine if they also produce field lines which are everywhere elevated. These shear functions are plotted in Figure 3 (note that $\gamma(x, 0)$ is specified only in the region of nonzero flux). The function labelled 2 is from Klimchuk, Sturrock, and Yang (1988), and the function labelled 4 is repeated from Figure 1 for comparison. All of the shear functions have an amplitude of 6 , which is comparable to the half- 
width of the arcade at its base and corresponds to a footpoint displacement of $\approx$ $6 \times 10^{4} \mathrm{~km}$ in a solar active region. Force-free field models were calculated for each of these shear functions using the same normal field boundary condition as before.

In each case all of the field lines are elevated compared with the potential field. This is not surprising in models 1,2 , and 3 , where $B_{z}$ decreases monotonically outward from the arcade axis, but in model $5, B_{z}$ is greatest along the $\alpha=0.67$ field line. The degree to which the field becomes inflated is indicated in Table 1, where we give the change in height along the $y$ axis $(x=0)$ of three representative field lines: an inner $(\alpha=0.8)$, a middle ( $\alpha=$ 0.5 ), and an outer ( $\alpha=0.2$ ) field line. As expected, the absolute height change increases as one moves outward from the arcade axis. We see that the magnitude of the change can be quite substantial. In model 1 , for example, the outer field line rises by an amount comparable to the width of the arcade itself. Still higher field lines rise by even more.

Also given in Table 1 , in parentheses, is the percentage change in height, $\Delta y / y$. We see that the heights of the inner and middle field lines have more than doubled in the first two models. The manner in which the percentage height change varies with $\mathrm{y}$ is somewhat different in the different models. In model 1 , where the shear profile is flat, $B_{z}$ decreases systematically outward according to equation (2.8), and so too does the fractional change in height. In models 4 and 5 , on the other hand, $B_{z}$ peaks midway between the inner and middle field lines, and we find that the fractional change in height of the middle field line is much greater than that of the inner field line. Thus, the field lines tend to spread apart preferentially in the region of greatest $B_{z}^{2}$ pressure, as one would expect. 
The models are ordered in Table 1 according to the energy of the field, $U$, given in the second to last column as the ratio $U / U_{0}$, where $U_{0}$ is the energy of the corresponding potential field (the same for all models). We find, not surprisingly, that the fields with the greatest energy are also the fields with the greatest inflation. In the last column of Table 1 we give the ratio $U / U_{0}$ that is predicted by a simple formula derived in Klimchuk, Sturrock, and Yang (1988) and thought to apply to a wide range of arcade configurations. The agreement between the predicted and actual values appears quite satisfactory.

Table 2 indicates how the inflation of a field varies with shear amplitude when the functional form of the shear is held fixed. All of the models were computed using the shear function

$$
\gamma(x, 0)=Z_{\max } \sin \left(\frac{\pi x}{8}\right)\left|\sin \left(\frac{\pi x}{8}\right)\right|
$$

and differ only in the value of $\mathrm{Z}_{\max }$ (from Klimchuk, Sturrock, and Yang 1988). The case $Z_{\max }=6$ is identical to model 2 in Figure 3 and Table 1 . As expected, the field becomes progressively more inflated as the amplitude of the shear is increased. The rate of inflation is faster than the rate of energy increase in the sense that the fractional change in height of the field lines is roughly twice the fractional change in energy of the field.

\section{MODEL OF LOW AND NAKÄGAWA}

Our inability to generate a model for which any of the field lines descend has prompted us to reexamine the model of Low and Nakagawa (1975), which is thought to exhibit this behavior. The boundary condition on $\alpha$ in that model is 


$$
\alpha(x, 0)=\cos x
$$

Low and Nakagawa take the generating function approach and specify

$$
\mathrm{B}_{\mathrm{z}}(\alpha)=-\varepsilon \frac{\alpha^{2}}{1+\alpha^{2}}
$$

where $\varepsilon$ is a parameter analogous to $Z_{\max }$. Since this particular form of the generating function is not amenable to analytic solution, the authors obtain a numerical solution over the domain $-\pi / 2 \leq \mathrm{x} \leq \pi / 2,0 \leq \mathrm{y} \leq 10$, where it is assumed that $\alpha=0$ on the outer boundary.

At this point we note an important property of equation (4.2): $\left|\mathrm{B}_{\mathrm{z}}\right|$ is an increasing function of $\alpha$ and therefore a decreasing function of $|\mathbf{x}|$ throughout the domain of the solution. The pressure gradient force associated with $B_{z}$ is everywhere outward. For this reason the apparent result of Low and Nakagawa that the field becomes depressed is suspicious.

In order to reconstruct the Low and Nakagawa field using the magnetofrictional method we must know the z-separation of each pair of footpoints (the shear function). This can be obtained from the generating function solution by integrating along field lines according to equation (2.7), as was done by Low and Nakagawa for five representative field lines. The resulting values for the case $\varepsilon=0.2$ are plotted in Figure 4 (taken from Figure 3 in Low and Nakagawa). In addition, we know that the outermost field line intersecting the photosphere at $x= \pm \pi / 2$ has no shear, because $B_{z}=0$ for this field line. Finally, while we have no detailed information on the footpoint separation 
near the arcade axis (this was not calculated by Low and Nakagawa), we do know that the $B_{z}$ pressure will be smallest for a small separation. In order to maximize the tendency for the field to become depressed, we therefore take the footpoint separation to vanish at $x=0$. A least-squares fit of the resulting 7 data points leads to the following polynomial expression for the shear function:

$$
\gamma(x, 0)=c_{4} x^{4}+c_{3} x^{3}+c_{2} x^{2}+c_{1} x
$$

where $c_{4}=-1.154, c_{3}=2.829, c_{2}=-2.543, c_{1}=1.487$. This shear function is plotted in Figure 4.

We have computed a force-free field by the magneto-frictional method using equations (4.1) and (4.3) as photospheric boundary conditions and using the same computational domain and outer boundary conditions as were used by Low and Nakagawa. The field is shown in Figure 5 together with the corresponding potential field $[\gamma(x, 0)=0]$. The sheared field is clearly inflated compared with the potential field, as expected on the basis of the above discussion and in direct contradiction with the generating function result of Low and Nakagawa. We conclude that the earlier result is either incorrect or else has a topology that is more complicated than originally thought, a conclusion that is shared by Low (private communication).

It is a well-known property of generating function solutions that they are not always unique (e.g., Birn, Goldstein, and Schindler 1978; Low 1982; Heyvaerts et al. 1982). Depending on the form of the generating function, there may be two or more solutions which satisfy the same boundary condition on $\alpha(x, 0)$ and have the same $B_{z}(\alpha)$ dependence, but which have a different field line connectivity (i.e., footpoint shear). This is true of the generating function used by Low and Nakagawa, for example. It is also possible to have multiple 
solutions which satisfy the same boundary condition on $\alpha(x, 0)$ and have the same field line connectivity, but which have a different $B_{z}(\alpha)$ dependence (Klimchuk and Sturrock 1989; see also Priest and Milne 1980). Low and Nakagawa attempted to find the solution having the simplest topology--the one for which all of the field lines are connected to the photosphere. It may be, however, that they instead found a solution with disconnected or "floating" flux (Low, private communication). If the quantity of floating flux increases with $\varepsilon$, as it does in the generating function sequence of Low (1977), the magnetic pressure associated with the flux might force the underlying, arcade field downward, in agreement with the Low and Nakagawa result.

\section{DISCUSSION}

The results of the previous two sections lead us to propose the following conjecture: In fields having a simple arcade geometry (without floating flux), any form of footpoint shear, even one that is localized away from the arcade axis, will cause all of the field lines to rise and the entire configuration to inflate. Moreover, by equation (2.5), any enhancement to thermal gas pressure must have the same effect on magnetostatic fields for which the pressure does not depend explicitly on height. Although we have been unable to prove this as a general theorem, we are able to provide two plausibility arguments.

First, Aly (1985) has shown that as the footpoint shearing displacements of an arcade field become arbitrarily large, the field approaches the "open field" configuration as its limiting state (although see Finn and Chen 1989). For any shear function $Z_{\max } \gamma(x)$ that is everywhere nonzero, all of the field lines extend to infinity as $Z_{\max } \rightarrow \infty$. In other words, the field becomes infinitely inflated. It is reasonable to expect that a sheared arcade will monotonically approach this limiting state, rather than first deflating for 
some range of shear and then reversing direction and inflating for larger values of shear. We see no reason for why the interplay between magnetic tension and pressure gradient forces should behave differently for small and large values of shear.

As a second plausibility argument we invoke a simple hydrostatic analogy. Consider a semi-infinite vertical tube filled with an isothermal, gravitationally stratified gas. The tube is capped at the bottom, but not at the top. Suppose that the temperature of the particles in some layer part way up the tube is increased, as indicated in Figure 6. If the gas responds adiabatically to the temperature increase, a new equilibrium will be reached in which the hotter gas and the cooler gas above are elevated, but the cooler gas below is unchanged (for uniform gravity). This result comes straightforwardly from the fact that the pressure at any point in the gas must be equal to the weight of the overlying material:

$$
P(y)=\int_{y}^{\infty} \rho g d y .
$$

The weight of the material is of course unaffected by the temperature increase. Initially there will be a pressure increase in the heated layer, and no doubt the transient response will be to force the underlying material downward, but eventually the atmosphere will adjust such that none of the material is depressed in the final static equilibrium. In fact, if gravity decreases with height, as it does in the solar atmosphere, all of the material will become elevated compared with its original position, including the material that lies below the heated layer (as pointed out by P. A. Sturrock). 
This result is analogous to our result for magnetic fields in which all of the field lines rise, even when the $\mathrm{B}_{\mathrm{z}}{ }^{2}$ magnetic pressure is confined to a subset, or layer, of field lines. Also note that the thickness of the heated layer will increase, just as the field lines in model 4 (Figure 2) were found to spread preferentially in the layer of shear. In both cases the effect of the expansion is to alleviate the pressure increase brought about by the imposed changes:- in the magnetic problem, a decrease in $\mathrm{Bx}_{\mathrm{x}}^{2}+\mathrm{B}_{\mathrm{y}}{ }^{2}$ tends to offset the pressure increase due to $\mathrm{B}_{\mathrm{z}}{ }^{2}$, while in the hydrostatic problem, a decrease in particle density offsets the pressure increase due to the increase in temperature. An important point is that the expansion is entirely upward. There is no external boundary to inhibit this upward expansion, whereas a downward expansion is prevented by the rigid photosphere and the cap on the bottom of the tube.

In hydrostatic equilibrium, thermal pressure gradients are balanced by gravitional forces, while in a force-free magnetic field, magnetic pressure gradients are balanced by magnetic tension forces. Analogous to equation (5.1) therefore is the result that the magnetic pressure at any point along the $\mathrm{y}$-axis of a force-free arcade is equal to the integrated effect of the magnetic tension in the overlying field:

$$
\frac{1}{8 \pi} B(y)^{2}=-\frac{1}{4 \pi} \int_{y}^{\infty} B_{x} \frac{\partial B_{y}}{\partial x} d y .
$$

If all the field lines of an arcade rise, then the field strength and magnetic pressure at the arcade axis $(y=0)$ must certainly decrease (for $B_{z}=0$ at the axis), and so too must the total magnetic tension force integrated along the $y$ axis from zero to infinity. 
In Figure $7 \mathrm{~b}$ we show the integrated magnetic tension forces in the unsheared arcade of Figure $2 a$ and the sheared arcade of Figure $2 b$, integrated along the $y$-axis between the limits of 0 and $y$, and plotted as a function of $y$. The two curves indicate the cumulative effect of magnetic tension as one move upward along the y-axis from the photosphere. The curves clearly diverge, with the curve for the sheared (inflated) field falling below that of the unsheared field, as expected from equation (5.2). At the upper boundary of the model $(y=60)$, the tension integrals differ by $17.04 \%$. This agrees very closely with the $17.22 \%$ difference in magnetic pressures at the arcade axis, indicating the high degree of accuracy of the models and the unimportance of the far-removed upper boundary. In models where the upper boundary is important, the nonzero pressure at that boundary must be added to the righthand-side of equation (5.2) (or equation [5.1]). It is possible for the inner part of a sheared field to become depressed in such a model, although this does not seem to be the problem with the Low and Nakagawa result.

By comparing Figures $7 \mathrm{a}$ and $7 \mathrm{~b}$, we see that the reduction in magnetic tension in the sheared arcade occurs mostly in the region of greatest shear, where $B_{Z}$ is large. We can express the tension force as

$$
F_{\text {tension }}=\frac{1}{4 \pi} \frac{B^{2}}{R}
$$

where $R$ is the local radius of curvature, given by

$$
R(x=0)=-\frac{B_{x}^{2}+B_{z}^{2}}{B_{x}}\left(\frac{d B_{y}}{d x}\right)^{-1}
$$


along the y-axis. Since the tension force is reduced in the region of shear, we know that the magnetic pressure gradient must also be reduced in order to have force balance. This suggests that $\mathrm{B}^{2}$ will be relatively large in the region of shear, or at least comparable to its potential field value, which we have confirmed to be the case. The significant reduction in magnetic tension must therefore be due to an increase in the radius of curvature.

In Figure $7 \mathrm{c}$ we have plotted the radius of curvature as a function of height along the $y$-axis. We see that, indeed, the radius of curvature is increased in the region of shear, by up to a factor of two. This "flattening" of the field lines must be due entirely to their being stretched in the z-direction, since the field lines also become elevated, which tends to decrease their radius of curvature. The latter effect is especially evident for the lower lying, weakly sheared field lines, for which the radius of curvature actually decreases. We note that the radius of curvature of the outermost field lines is probably affected by the upper boundary and should therefore be treated with caution.

\section{CONCLUSION}

In summary, we have conjectured that any footpoint shear imposed upon a simple arcade magnetic field will cause the entire field to become inflated--all of the field lines will rise. We have shown this to be true for a number of different shear profiles, including two which maximize $B_{z}$ a finite distance away from the arcade axis. Field lines tend to spread apart in the region of enhanced $B_{z}$ (as also found by Amari et al. 1989), but all of the field lines rise--the higher field lines simply rise more than the lower field lines. We have also demonstrated that the original depressed field result of Low and Nakagawa (1975) is incorrect, and that the sheared field actually becomes inflated. 
We note that field lines can become temporarily depressed in response to a sudden or rapidly changing shear (rapid compared to the characteristic Alfven travel time) (e.g., Wu et al. 1983), but this is a property of configurations that are not in equilibrium, and we are here concerned with configurations that are evolving quasi-statically and at all times well-approximated by a static equilibrium.

Tables 2 and 3 show that the degree to which fields become inflated can be substantial, especially in the outer portions of the configuration. This suggests that the shearing of magnetic fields may be an important process leading to the opening of magnetic field lines. As closed field lines are brought to greater heights in the solar atmosphere, they are more likely to be opened up by the thermal pressure and dynamic forces of the plasma (e.g., Pneuman and Kopp 1971). Thus, we might expect shearing motions on the surface of the Sun to be associated with an increase of the interplanetary magnetic flux and possibly an increase in coronal hole area.

Finally, we have shown that, although field lines rise, they can nonetheless by flatted by the stretching due to the shearing displacement. This has important consequences for the formation of solar prominences, since prominences cannot form along field lines that are well-rounded (i.e., with a small radius of curvature) and concave downward (see the discussions in Priest, Hood, and Anzer 1989 and Amari et al. 1989). We find no evidence for a dip in the field--a region of upward concavity--in agreement with the recent result of Amari et al. (1989).

We note that low-lying, relatively flat field lines occur naturally above "weak field corridors" of sharply reduced flux (see Figures 2 and 7c), in agreement with observation (Klimchuk 1987, 1988). It is in these corridors that active region filaments are found to occur (e.g., Klimchuk 1986, 1987). The 
degree of flatness of the field lines can be increased by shearing their footpoints, near the edge of the corridor, and indeed corridor fields and filaments are often observed to have a high degree of shear (e.g., Klimchuk 1987; Zirin 1972). It is not surprising, therefore, that active region filaments occur preferentially in weak field corridors where the field is highly sheared.

It is a great pleasure to acknowledge many useful discussions with my colleagues Peter A. Sturrock and Spiro K. Antiochos. This work was supported in part by Office of Naval Research contract N00014-85-K-0111, by NASA grant 05-020-272, and as part of the Solar-A collaboration under NASA contract NAS8-37334 with Lockheed Palo Alto Research Laboratories. The numerical calculations were performed on the Cray X-MP of the San Diego Supercomputer Consortium. 
TABLE 1

FIELD LINE ELEVATION RELATIVE TO THE POTENTIAL FIELD

\begin{tabular}{crrrrr} 
Model & $(\alpha=0.8)$ & $(\alpha=0.5)$ & $\begin{array}{c}\Delta y \\
(\alpha=0.2)\end{array}$ & $\begin{array}{c}\text { Actual } \\
\mathrm{U} / \mathrm{U}_{0}\end{array}$ & $\begin{array}{c}\text { Predicted } \\
\mathrm{U} / \mathrm{U}_{0}\end{array}$ \\
\hline 1 & 3.19 & 6.26 & 12.47 & 1.96 & 1.87 \\
& $(219 \%)$ & $(171 \%)$ & $(107 \%)$ & & \\
2 & 1.18 & 4.07 & 8.60 & 1.55 & 1.51 \\
& $(107 \%)$ & $(111 \%)$ & $(74 \%)$ & & \\
3 & 0.45 & 1.50 & 4.49 & 1.24 & 1.26 \\
& $(41 \%)$ & $(41 \%)$ & $(38 \%)$ & & \\
4 & 0.11 & 1.85 & 4.05 & 1.20 & 1.22 \\
& $(10 \%)$ & $(50 \%)$ & $(35 \%)$ & & \\
& 0.05 & 0.49 & 2.46 & 1.07 & 1.13
\end{tabular}

TABLE 2

FIELD LINE ELEVATION RELATIVE TO THE POTENTIAL FIELD: EQUATION (3.6) SHEAR FUNCTION (MODEL 4)

\begin{tabular}{crrrrr}
$\mathrm{Z}_{\max }$ & $\begin{array}{r}\Delta \mathrm{y} \\
(\alpha=0.8)\end{array}$ & $\begin{array}{c}\Delta \mathrm{y} \\
(\alpha=0.5)\end{array}$ & $\begin{array}{c}\Delta \mathrm{y} \\
(\alpha=0.2)\end{array}$ & $\begin{array}{c}\text { Actual } \\
\mathrm{U} / \mathrm{U}_{0}\end{array}$ & $\begin{array}{c}\text { Predicted } \\
\mathrm{U} / \mathrm{U}_{0}\end{array}$ \\
\hline 2.0 & 0.15 & 0.55 & 1.26 & 1.09 & 1.08 \\
& $(14 \%)$ & $(15 \%)$ & $(11 \%)$ & & \\
6.0 & 1.18 & 4.07 & 8.60 & 1.55 & 1.51 \\
& $(107 \%)$ & $(111 \%)$ & $(74 \%)$ & & \\
10.0 & 2.84 & 8.86 & 16.64 & 2.03 & 1.99 \\
& $(258 \%)$ & $(241 \%)$ & $(142 \%)$ & &
\end{tabular}




\section{REFERENCES}

Aly, J. J. 1985, Astr. Ap., 143, 19.

Amari, T., Demoulin, P., Browning, P., Hood, A., and Priest, E. 1989, Astr. Ap., submitted.

Barnes, C. W., and Sturrock, P. A. 1972, Ap. J., 174, 659.

Birn, J., Goldstein, H., and Schindler, K. 1978, Solar Phys., 57, 81.

Biskamp, D., and Welter, H. 1989, Solar Phys., 120, 49.

Chodura, R., and Schluter, A. 1981, J. Comp. Phys., 41, 68.

Craig, I. J. D., and Sneyd, A. D. 1986, Ap. J., 311, 451.

Finn, J. M., and Chen, J. 1989, Ap. J., in press.

Heyvaerts, J., Lasry, J. M., Schatzman, M., and Witomsky, P. 1982, Astr. Ap., $111,104$.

Hundhausen, J. R., Hundhausen, A. J., and Zweibel, E. G. 1981, J. Geophys. Res., 86, 1117.

Jockers, K. 1978, Solar Phys., 56, 37. 
Klimchuk, J. A. 1986, in A. I. Poland (ed.), Coronal and Prominence Plasmas, NASA CP-2442, p. 183. 1987, Ap. J., 323, 368.

. 1988, Solar Phys., 119, 19. 1989, Bull. A. A. S., 21, 864.

Klimchuk, J. A., and Sturrock, P. A. 1989, Ap. J., in press.

Klimchuk, J. A., Sturrock, P. A., and Yang, W.-H. 1988, Ap. J., 335, 456.

Low, B. C. 1977, Ap. J., 212, 234. . 1982, Rev. Geophys. and Sp. Phys., 20, 145.

Low, B. C., and Nakagawa, Y. 1975, Ap. J., 199, 237.

Mikic, Z., Barnes, D. C., Schnack, D. D. 1988, Ap. J., 328, 830.

Pneuman, G. W., and Kopp, R. A. 1971, Solar Phys., 18, 258.

Poland, A. I., Mariska, J. T., and Klimchuk, J. A. 1986, in A. I. Poland (ed.), Coronal and Prominence Plasmas, NASA CP-2442, p. 57.

Priest, E. R. 1982a, Solar Magnetohydrodynamics (Dordrecht: Reidel). 
1982b, Fundamentals of Cosmic Physics, 7, 363.

Priest, E. R., and Milne, A. M. 1980, Solar Phys., 65, 315.

Priest, E. R., Hood, A. W., and Anzer, U. 1989, Ap. J., submitted.

Sheeley, N. R., Jr., Nash, A. G., and Wang, Y.-M. 1987, Ap. J., 319, 481.

Sturrock, P. A., and Woodbury, E. T. 1967, in Plasma Astrophysics, ed. P. A. Sturrock (New York: Academic), p. 155.

Wang, Y.-M., and Sheeley, N. R., Jr. 1989, Ap. J., in press.

Wolfson, R., and Gould, S. A. 1985, Ap. J., 296, 287.

Wu, S. T., Hu, Y. Q., Nakagawa, Y., and Tandberg-Hanssen, E. 1983, Ap. J., $266,866$.

Yang, W.-H., Sturrock, P. A., and Antiochos, S. K. 1986, Ap. J., 309, 383.

Zirin, H. 1972, Solar Phys., 22, 34.

Zwingmann, W. 1987, Solar Phys., 111, 309. 


\section{FIGURE CAPTIONS}

Fig. 1.--Distributions of the normal component of the photospheric magnetic field, $B_{y}(y=0)$, given by equation (3.3) and the footpoint $z$-displacement, $\gamma(y=0)$, given by equation (3.5), plotted as a function of distance from the arcade axis. The distributions are antisymmetric about the axis, and only the positive $x-$ halves are shown. Plotted points indicate the spatial resolution of the model calculations. These boundary conditions correspond to model 4 in Table 1 .

Fig. 2.--Contours of constant $\alpha$ for models having photospheric boundary conditions given by equations (3.4) and (3.5): (a) the potential field case $\mathrm{Z}_{\max }=$ 0 ; (b) the sheared case $Z_{\max }=6$ (model 4). These plots represent end-on views of the arcades. Please note that the domain of the calculations $(60 \times 60)$ is much larger than shown.

Fig. 3.--Shear functions showing the distribution of footpoint z-displacements as a function of distance from the arcade axis. The functions are antisymmetric about the axis, and only the positive $x$-halves are shown. Plotted points indicate the spatial resolution of the model calculations. Number labels refer to model numbers in Table 1.

Fig. 4.--Adopted shear function for our computation of the Low and Nakagawa model (Case II). Points are from Figure 3 of Low and Nakagawa (1975).

Fig. 5.--Contours of constant $\alpha$ for our computation of the Low and Nakagawa model. The boundary conditions on $\alpha$ are given by equation (4.1) and the 
boundary conditions on $\gamma$ are given by: (a) $\gamma(x, 0)=0$ (the potential field); or (b) equation (4.3).

Fig. 6.--Hydrostatic analogy to the sheared force-free magnetic field problem: (a) semi-infinite vertical tube filled with an isothermal, gravitationally stratified gas; (b) resulting equilibrium after the gas has responded adiabatically to a temperature increase of the particles in some layer (gravity is assumed to decrease with height).

Fig. 7.--Magnetic field quantities plotted as a function of position along the $y$ axis (height) for the unsheared arcade of Figure 2a (dashed curves) and the sheared arcade of Figure $2 b$ (solid curves): (a) $B_{z}$ component of the field; (b) cumulative magnetic tension force integrated from the base $(y=0)$ to height $y$; (c) local radius of curvature of the field. The units of (a) and (b) are arbitrary. 


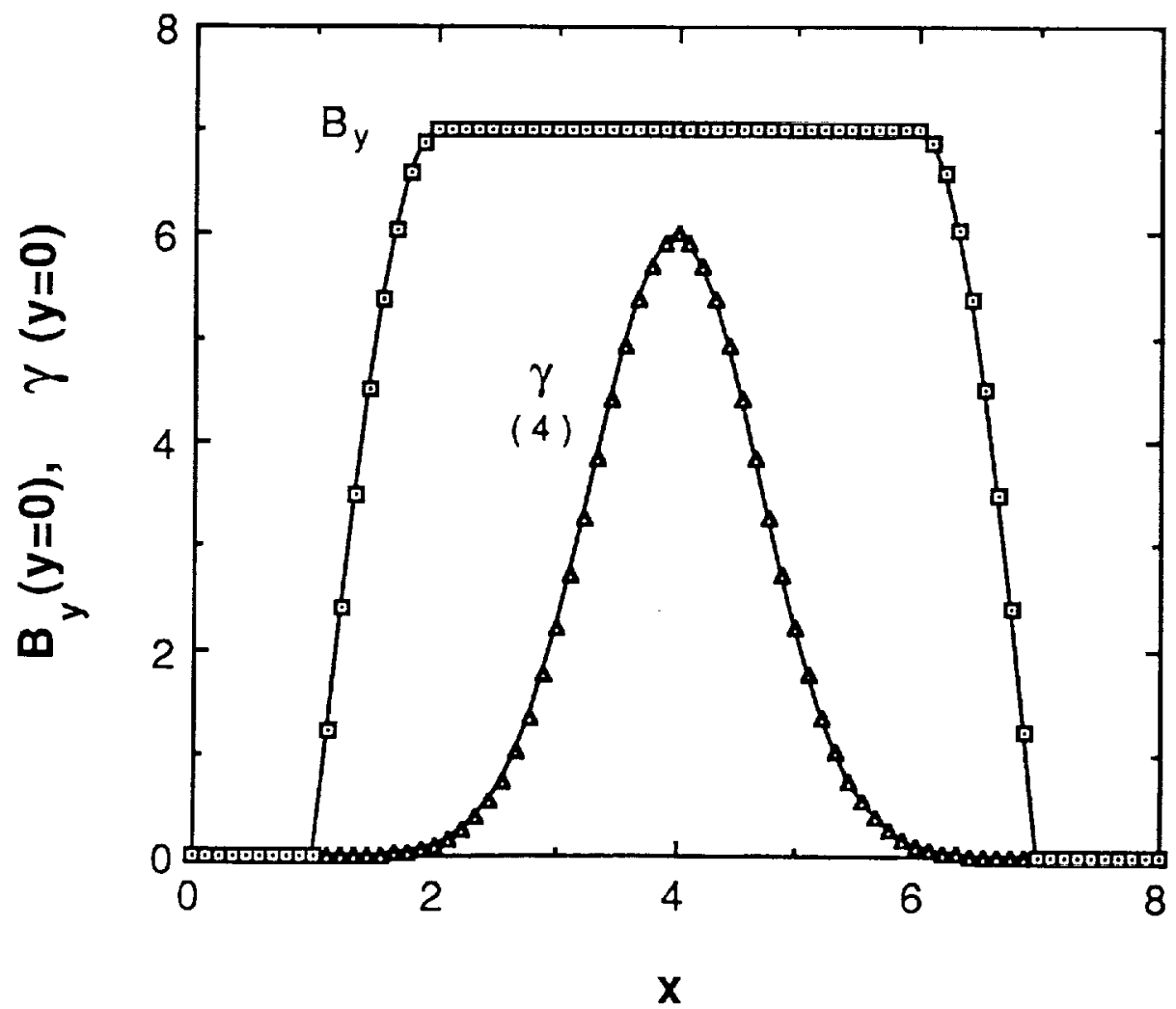

Figure 1. 


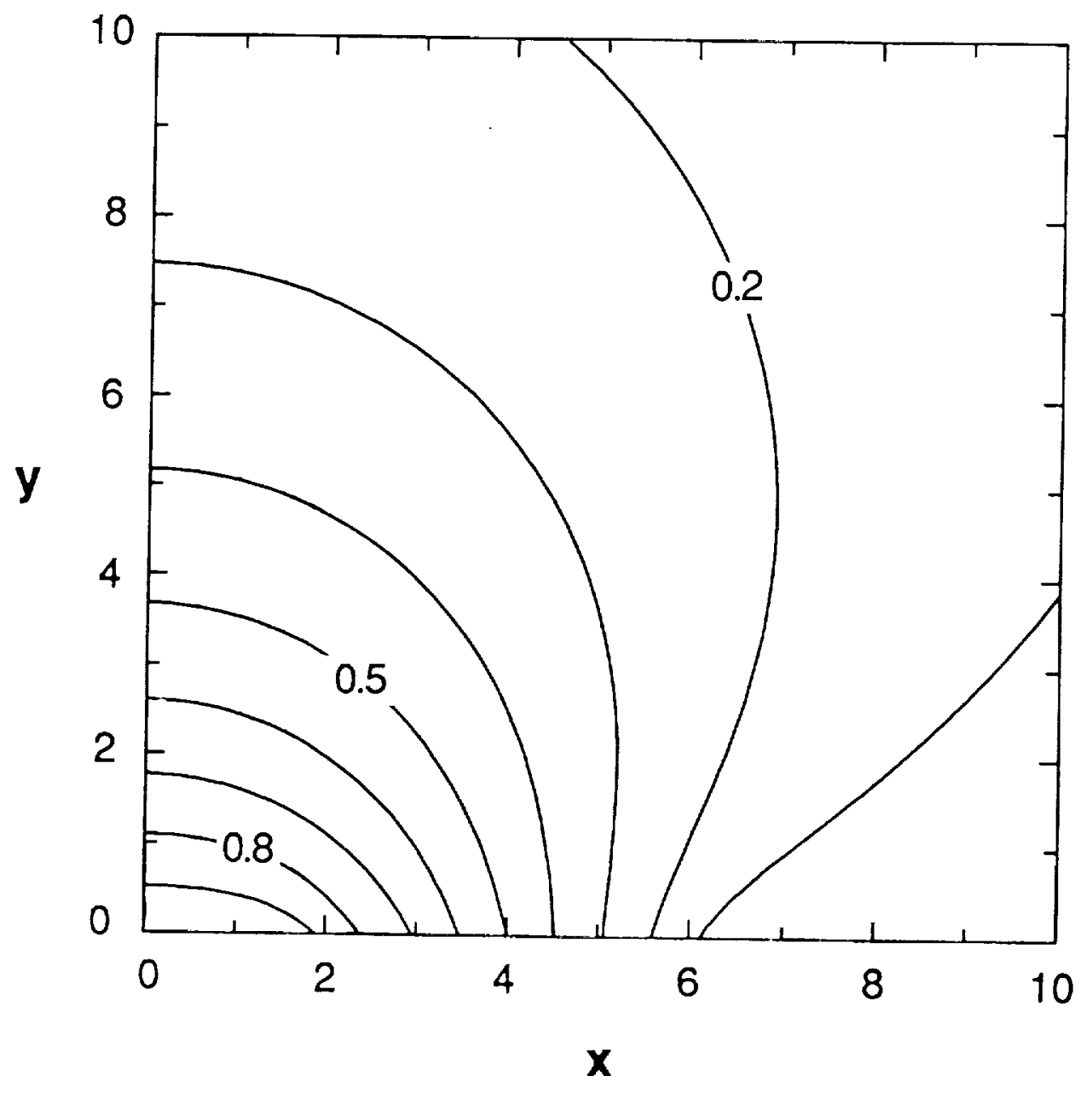

(a)

Figure 2. 



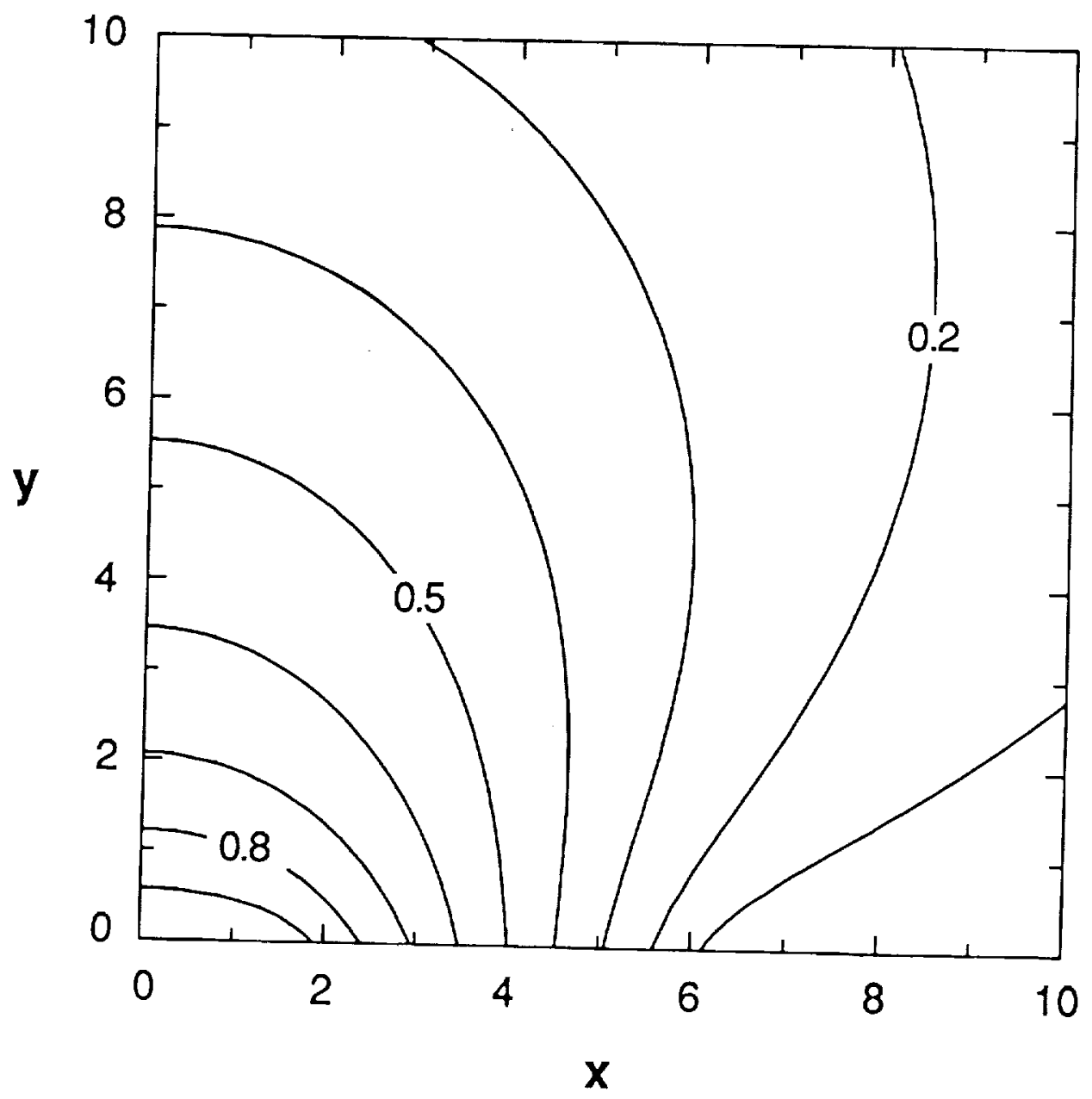

(b)

Figure 2.: $A$ 


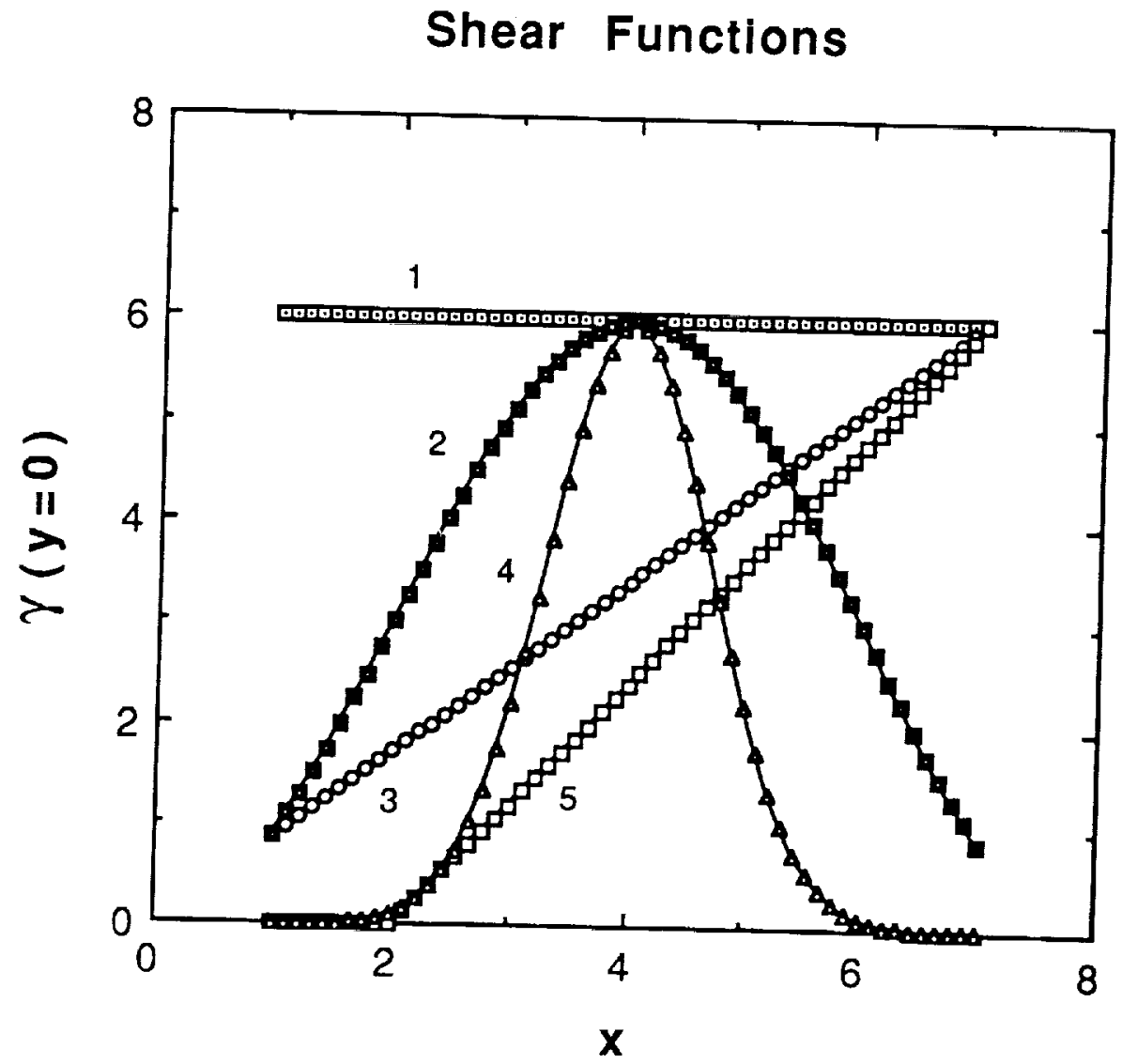

Figure 3. 
. 


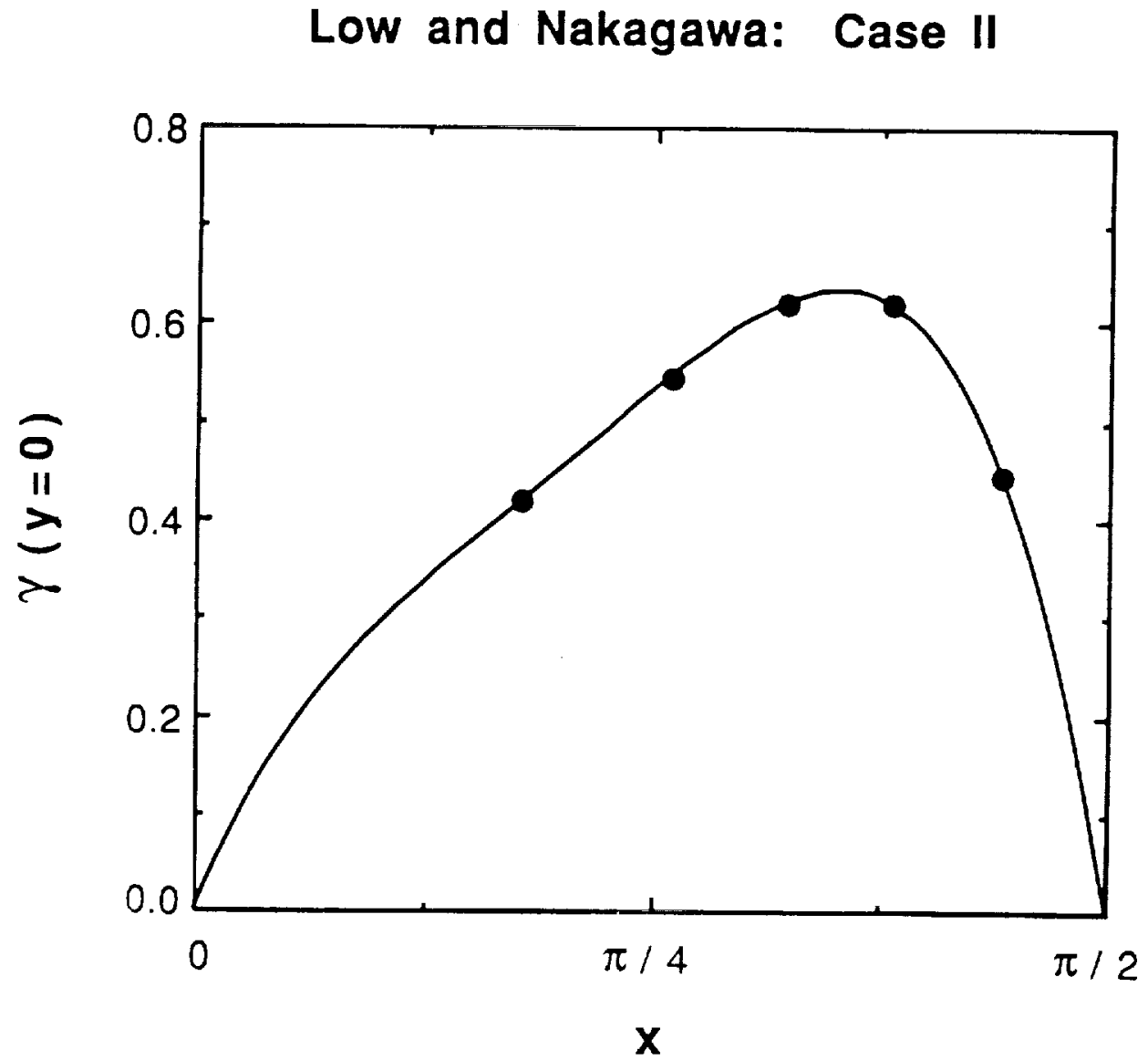

Figure 4. 


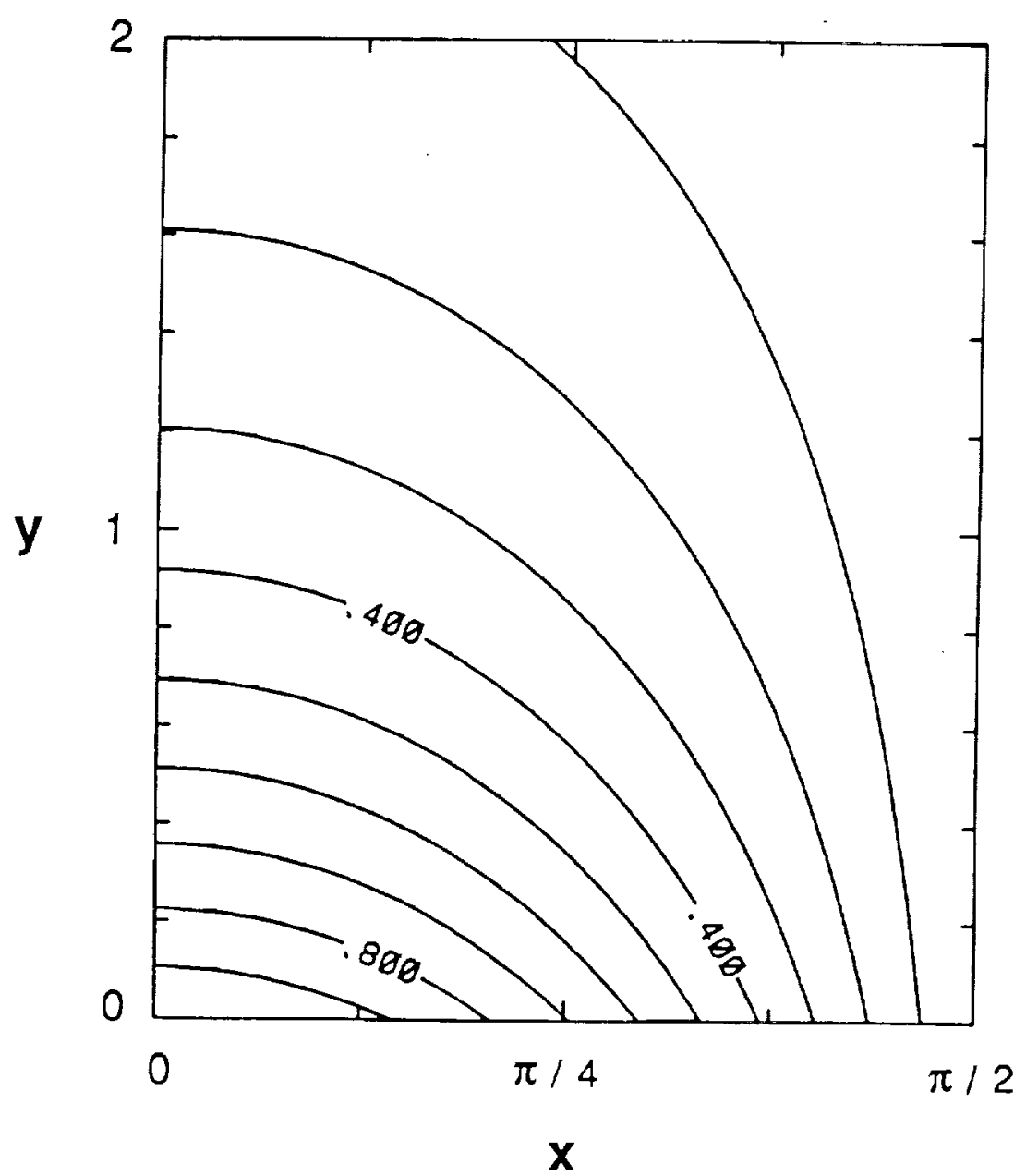

(a)

Figure 5. 


\author{
•
}

\author{
.
}




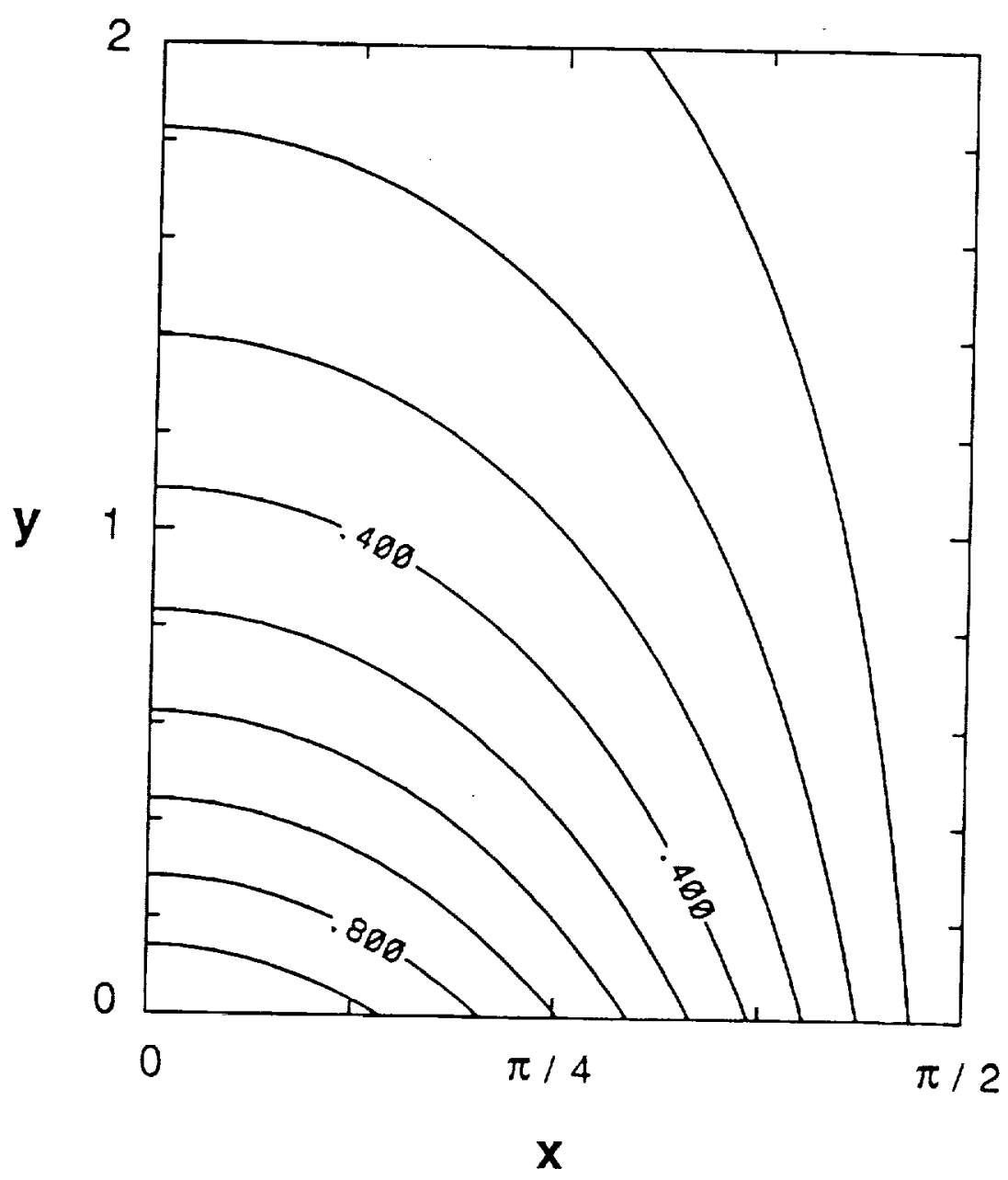

(b)

Figure 5.:A 



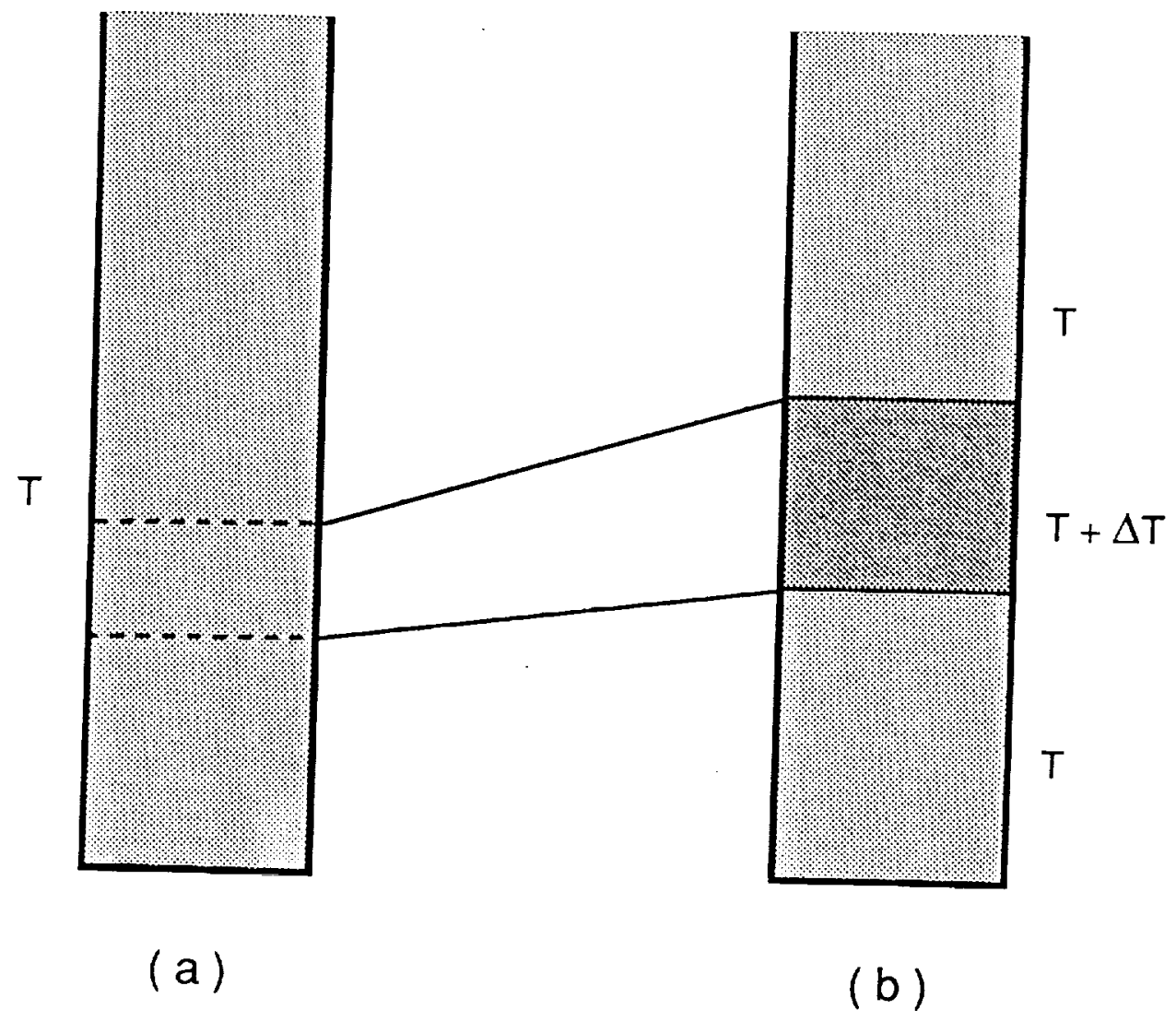

Figure 6 . 



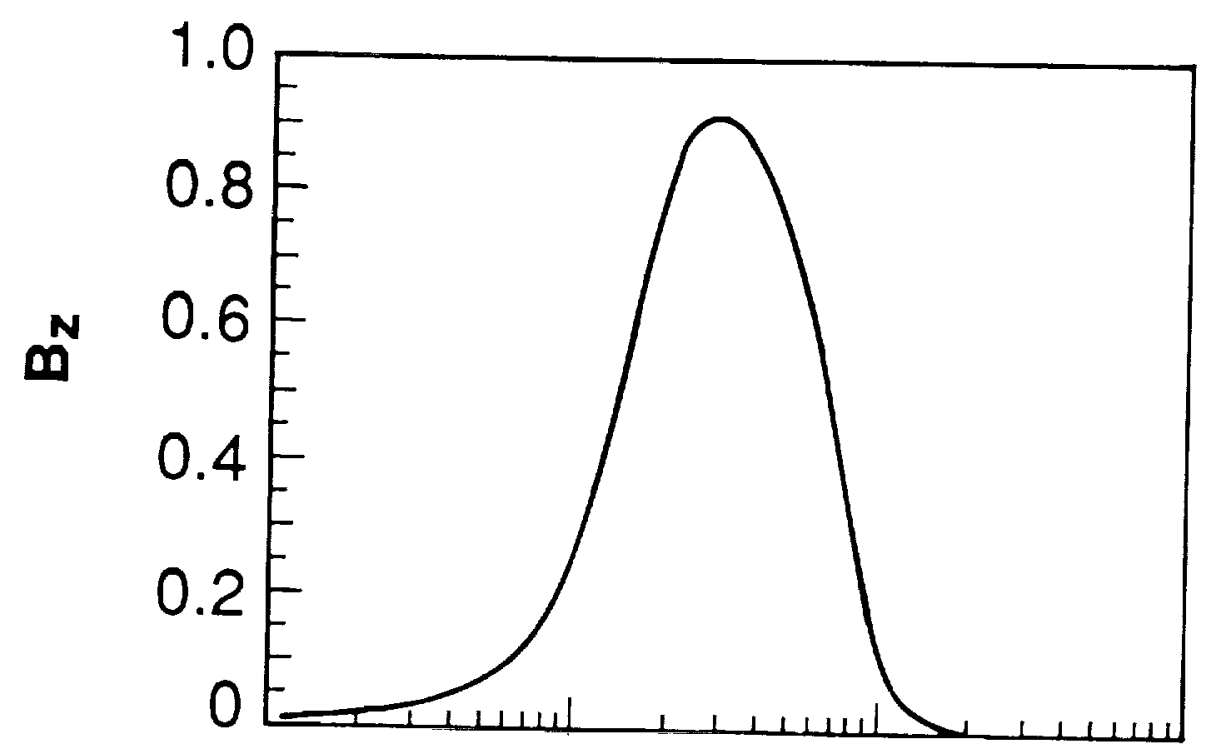

(a)

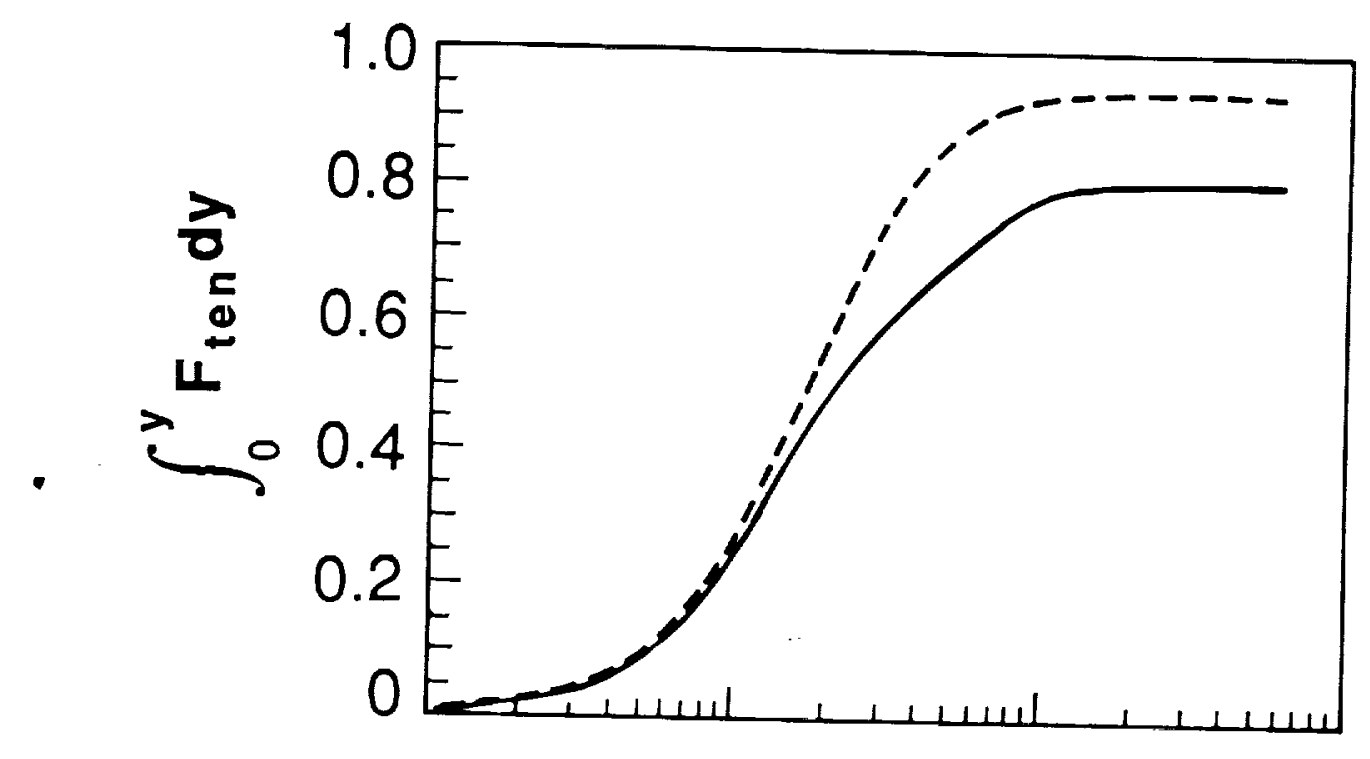

(b)

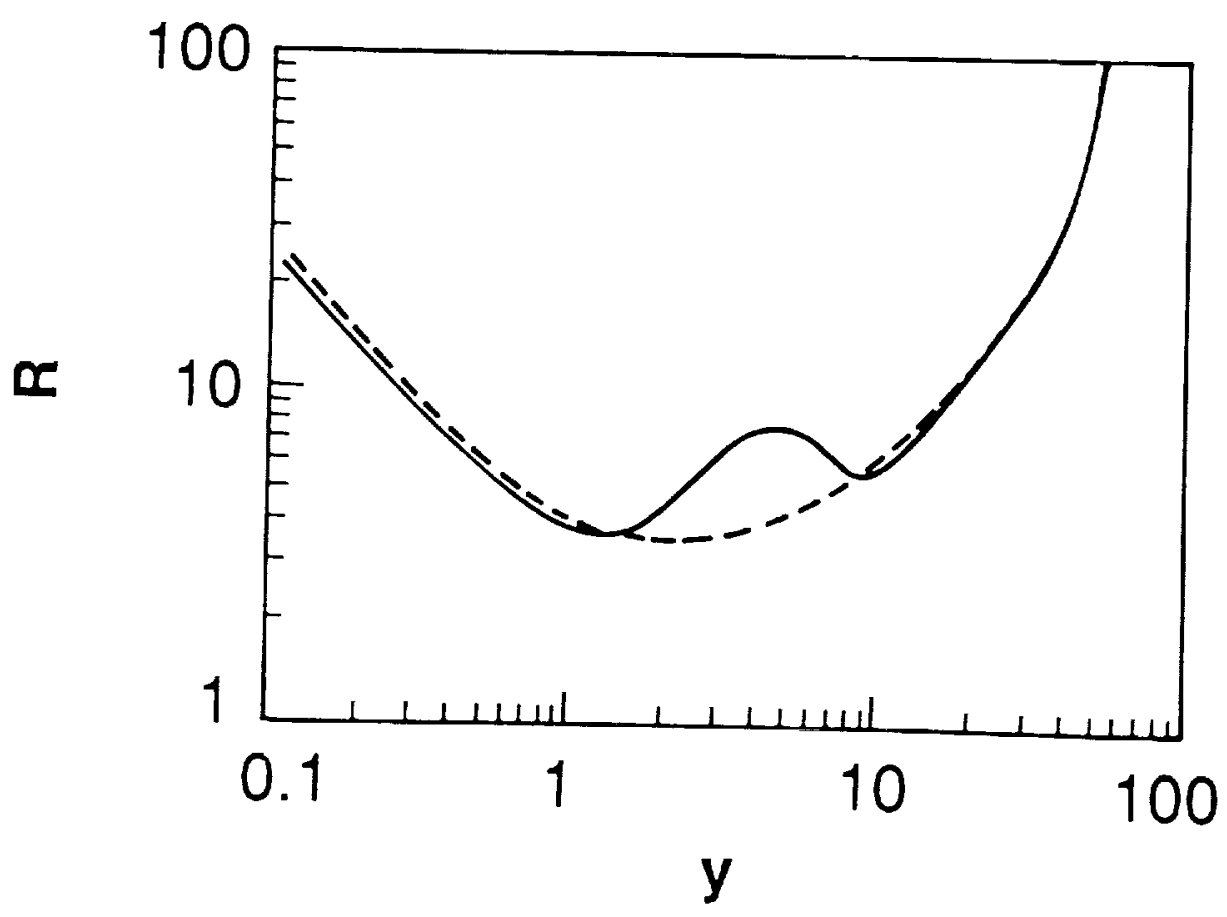

(c)

Figure 7. 



\section{ADDRESS:}

James A. Klimchuk

Center for Space Science and Astrophysics

Stanford University, ERL 300

Stanford, CA 94305 
. 